Georgian Mathematical Journal

Volume 8 (2001), Number 4, 683-696

\title{
SUSPENSION AND LOOP OBJECTS AND REPRESENTABILITY OF TRACKS
}

\author{
H.-J. BAUES AND M. JIBLADZE
}

\begin{abstract}
In the general setting of groupoid enriched categories, notions of suspender and looper of a map are introduced, formalizing a generalization of the classical homotopy-theoretic notions of suspension and loop space. The formalism enables subtle analysis of these constructs. In particular, it is shown that the suspender of a principal coaction splits as a coproduct. This result leads to the notion of theories with suspension and to the cohomological classification of certain groupoid enriched categories.
\end{abstract}

2000 Mathematics Subject Classification: 18D05, 55P35, 55P40.

Key words and phrases: Groupoid enriched category, suspension object, loop object, suspender, looper, principal coaction.

A category enriched in groupoids (termed a track category for short) is a special 2-category. A track category $\mathscr{T}$ consists of objects $A, B, \ldots$ and homgroupoids $\llbracket A, B \rrbracket$ in which the objects are maps (1-arrows or 1-cells) and the morphisms are isomorphisms termed tracks (2-arrows or 2-cells). For each map $f: A \rightarrow B$ in $\mathscr{T}$ we have the group $\operatorname{Aut}(f)$ consisting of all tracks $\alpha: f \Rightarrow f$ in $\mathscr{T}$. This is an automorphism group in the hom-groupoid $\llbracket A, B \rrbracket$.

Our leading example is the track category Top* consisting of spaces $A, B, \ldots$ with basepoint ${ }^{*}$, pointed maps $f: A \rightarrow B$ and tracks $\alpha: f \Rightarrow g$ which are homotopy classes (relative to the boundary) of homotopies $f \simeq g$; compare (1.3) in [5]. For the trivial map $0: A \rightarrow * \rightarrow B$ in Top* one has the well known isomorphism of groups

$$
\operatorname{Aut}(A \rightarrow * \rightarrow B)=[\Sigma A, B] .
$$

Here the left-hand side is the group of automorphisms of $0: A \rightarrow B$ in the track category Top* and the right-hand side is the group of homotopy classes of maps $\Sigma A \rightarrow B$ where $\Sigma A$ is the suspension of $A$. Dually we also have the canonical isomorphism

$$
\operatorname{Aut}(A \rightarrow * \rightarrow B)=[A, \Omega B],
$$

where $\Omega B$ is the loop space of $B$. Via $(*)$ and $(* *)$ certain tracks in the track category $\mathscr{T}=$ Top $^{*}$ are represented by morphisms in the homotopy category $\mathscr{T} \simeq$ of $\mathscr{T}$. We study in this paper the categorical aspects of such a representability of tracks which we call $\Sigma$-representability in $(*)$ and $\Omega$-representability in $(* *)$. For this we introduce the notion of suspender generalizing the notion of 
suspension above by means of a universal property. The categorical dual of a suspender is a looper in a track category generalizing the notion of loop space. A track category $\mathscr{T}$ is $\Sigma$-representable, resp. $\Omega$-representable, if suspenders, resp. loopers exist in $\mathscr{T}$. Of course the track category Top* of pointed spaces (more generally the track category associated to any Quillen model category) is both $\Sigma$-representable and $\Omega$-representable.

We describe basic properties of suspenders and loopers. In particular we show that the suspender of a principal coaction splits as a coproduct. This is a crucial result which leads to the notion of theories with suspension and the cohomological classification of certain $\Sigma$-representable track categories in [6].

In topology a typical example of a suspender of a pointed space $X$ is the space

$$
\Sigma_{*} X=S^{1} \times X / S^{1} \times\{*\} .
$$

Moreover a looper of $X$ is given by the free loop space

$$
\Omega_{*} X=\left(X^{S^{1}}, 0\right)
$$

with the function space topology and basepoint given by the trivial loop 0 . Splitting results $\Sigma_{*} X \simeq X \vee \Sigma X$ (resp. $\left.\Omega_{*} X \simeq X \times \Omega X\right)$ are well known in case $X$ is a co-H-group (resp. H-group). For example Barcus and Barratt [5] or Rutter [12] use implicitly the splitting to obtain basic rules of homotopy theory. This paper and its sequel [6] specifies the categorical background of some of these rules. Suspenders and loopers are also responsible for the properties of partial suspensions and partial loop operations discussed in [6]; compare also $[4,3,2]$.

The theory of $\Sigma$-representable track categories in this paper is also motivated by the approach of Gabriel and Zisman [7] who consider those properties of a track category $\mathscr{T}$ which imply existence of a Puppe sequence for mapping cones. The suspension $\Sigma A$ plays a crucial rôle in this sequence. Enriching the results in [7] we show that the main categorical nature of a suspension in a track category is described by the notion of suspender which is the link between tracks in $\mathscr{T}$ and homotopy classes of maps in $\mathscr{T}$. Such a link, for example, is needed in results of Hardie, Kamps and Kieboom [9, 8] and Hardie, Marcum and Oda [10] who study homotopy-theoretic secondary operations like Toda brackets in 2categories. This paper does not aim at combining the theory of exact sequences in homotopy theory as considered in [7] and the theory of suspenders since the notion of suspenders, resp. loopers, is quite sophisticated and new.

\section{1. -REPRESENTABle TRACK CATEGORIES}

We first introduce the following notation. In a groupoid $\mathbf{G}$ the composition of morphisms

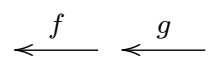


is denoted by $f+g$. Accordingly also the composition of tracks

$$
\stackrel{\alpha}{\longleftarrow} \stackrel{\beta}{\longleftarrow}
$$

in a track category is denoted by $\alpha+\beta$. We also write $\alpha: a \simeq b$ for a track $\alpha: a \Rightarrow b$. We say that a groupoid $\mathbf{G}$ is abelian if all automorphism groups of objects in $\mathbf{G}$ are abelian. For $\beta: y \rightarrow y$ and $\varphi: x \rightarrow y$ in $\mathbf{G}$ we obtain the conjugate

$$
\beta^{\varphi}=-\varphi+\beta+\varphi: x \rightarrow x
$$

so that $(-)^{\varphi}: \operatorname{Aut}(y) \rightarrow \operatorname{Aut}(x)$ is a homomorphism. The loop groupoid $\mathbf{G}$ of $\mathbf{G}$ is defined to have objects $\alpha: x \rightarrow x$, where $x$ is an object of $\mathbf{G}$; a morphism from $\alpha: x \rightarrow x$ to $\beta: y \rightarrow y$ is a morphism $\varphi: x \rightarrow y$ in $\mathbf{G}$ such that $\alpha=-\varphi+\beta+\varphi$.

Track functors between track categories and track transformations between track functors are the enriched versions of functors and natural transformations enriched in the category $\mathfrak{G} \mathfrak{p} \mathfrak{d}$ of groupoids. Here $\mathfrak{G} \mathfrak{p} \mathfrak{d}$ is also an example of a track category with functors between groupoids as 1-arrows and natural transformations as 2-arrows. Each object $C$ in a track category $\mathscr{T}$ yields the representable track functor

$$
\llbracket C,-\rrbracket: \mathscr{T} \rightarrow \mathfrak{G p \mathfrak { d }}
$$

between track categories which carries $X \in \mathrm{Ob}(\mathscr{T})$ to the hom-groupoid $\llbracket C, X \rrbracket$ in $\mathscr{T}$.

In a track category $\mathscr{T}$, consider a map $f: A \rightarrow B$. For any object $X$, denote by $\mathbf{G}_{f}(X)$ the following groupoid: objects of $\mathbf{G}_{f}(X)$ are pairs $(g, \alpha)$, where $g: B \rightarrow X$ is a map and $\alpha: g f \Rightarrow g f$ is a track. A morphism from $\left(g^{\prime}, \alpha^{\prime}\right)$ to $(g, \alpha)$ is a track $\gamma: g^{\prime} \Rightarrow g$ such that $\alpha^{\prime}=\alpha^{\gamma f}$. Any map $h: X \rightarrow Y$ induces a functor $\mathbf{G}_{f}(h): \mathbf{G}_{f}(X) \rightarrow \mathbf{G}_{f}(Y)$ sending $(g, \alpha)$ to $(h g, h \alpha)$ and $\gamma$ to $h \gamma$. Moreover any track $\eta: h \Rightarrow h^{\prime}$ induces a natural transformation $\mathbf{G}_{f}(\gamma): \mathbf{G}_{f}(h) \rightarrow \mathbf{G}_{f}\left(h^{\prime}\right)$ with components $\eta g: h g \Rightarrow h^{\prime} g$ for objects $(g, \alpha)$ of $\mathbf{G}_{f}(X)$. Thus we have defined a track functor

$$
\mathbf{G}_{f}: \mathscr{T} \rightarrow \mathfrak{G} \mathfrak{p d}
$$

Any object $(g: B \rightarrow C, \alpha: g f \Rightarrow g f)$ of $\mathbf{G}_{f}(C)$ gives rise to a track transformation

consisting of functors

$$
(g, \alpha)^{*}: \llbracket C,-\rrbracket \rightarrow \mathbf{G}_{f}
$$

$$
\llbracket C, X \rrbracket \rightarrow \mathbf{G}_{f}(X)
$$

which assign to $h: C \rightarrow X$ the pair $(h g, h \alpha)$ and to $\eta: h^{\prime} \Rightarrow h$ the track $\eta g$ (this indeed defines a morphism in $\mathbf{G}_{f}$ as $\eta g f+h^{\prime} \alpha=h \alpha+\eta g f$, i. e. $\left.h^{\prime} \alpha=(h \alpha)^{\eta g f}\right)$.

1.1. Definition. For a map $f: A \rightarrow B$ in a track category $\mathscr{T}$, a suspender for $f$ is a triple $\left(\Sigma_{f}, i_{f}, v_{f}\right)$ consisting of an object $\Sigma_{f}$, a map $i_{f}: B \rightarrow \Sigma_{f}$, and a track $v_{f}: i_{f} f \Rightarrow i_{f} f$ having the property that the induced track transformation

$$
\left(i_{f}, v_{f}\right)^{*}: \llbracket \Sigma_{f},-\rrbracket \rightarrow \mathbf{G}_{f}
$$

induces a bijection of isomorphism classes of objects. 
In other words, the following conditions must be satisfied:

(a) For any map $g: B \rightarrow C$ and any track $\eta: g f \Rightarrow g f$ there exists a map $\Sigma_{\eta}: \Sigma_{f} \rightarrow C$ and a track $\zeta_{\eta}: g \Rightarrow \Sigma_{\eta} i_{f}$ such that $\eta=\left(\Sigma_{\eta} v_{f}\right)^{\zeta_{\eta} f}$ (surjectivity);

(b) For any $h, h^{\prime}: \Sigma_{f} \rightarrow C$ and any track $\gamma: h^{\prime} i_{f} \Rightarrow h i_{f}$ with $h^{\prime} v_{f}=\left(h v_{f}\right)^{\gamma f}$ one has $\gamma=\delta i_{f}$ for some track $\delta: h^{\prime} \Rightarrow h$ (injectivity).

We point out that we do not assume for a suspender $\Sigma_{f}$ that the map $\left(i_{f}, v_{f}\right)^{*}$ is an equivalence of groupoids since this, in fact, does not hold in the example of topological spaces. Hence topology forces us to think of a weaker universal property, namely that $\left(i_{f}, v_{f}\right)^{*}$ induces only a bijection of isomorphism classes of objects. A track category $\mathscr{T}$ is $\Sigma$-representable if each map $f$ in $\mathscr{T}$ has a suspender $\left(\Sigma_{f}, i_{f}, v_{f}\right)$.

1.2. Definition. The dual notion of looper is obtained as a suspender in the opposite track category: a looper for $f: A \rightarrow B$ consists of a map $p_{f}: \Omega_{f} \rightarrow A$ and a track $\lambda_{f}: f p_{f} \Rightarrow f p_{f}$ satisfying conditions dual to the above ones for suspenders. A track category $\mathscr{T}$ is $\Omega$-representable if each map $f$ in $\mathscr{T}$ has a looper $\left(\Omega_{f}, p_{f}, \lambda_{f}\right)$.

Important particular cases are the suspenders and loopers for the identity map $1=\operatorname{id}_{A}: A \rightarrow A$ which will be denoted $\Sigma_{*}(A)$ and $\Omega_{*}(A)$ respectively; suspender for a map $0: A \rightarrow *$ to the initial object will be called suspension of $A$ and denoted $\Sigma_{0}(A)$, or simply $\Sigma(A)$ if the map 0 is uniquely determined by the context; and dually the looper for a map $0: 1 \rightarrow A$ from the terminal object to $A$ will be called loop object of $A$ and denoted $\Omega_{0}(A)$ or $\Omega(A)$.

These examples are important in that sometimes suspenders or loopers of all maps can be constructed using solely $\Sigma_{*}$ and $\Omega_{*}$ - indeed sometimes just using $\Sigma_{0}$ and $\Omega_{0}$. See below.

We consider the following examples of $\Omega$-representable and $\Sigma$-representable track categories.

1.3. Example. The track category $\mathfrak{G} \mathfrak{p d}$ of groupoids is $\Omega$-representable. In fact, for a functor $F: \mathbf{G} \rightarrow \mathbf{H}$ between groupoids the looper $\Omega_{F}$ is obtained by the pullback diagram

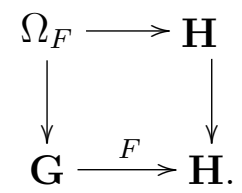

Moreover the loop groupoid $\mathbf{H}$ itself has the universal property for $\Omega_{*}(\mathbf{H})$.

1.4. Example. The track category Top* of pointed topological spaces is $\Sigma$ representable. Let $I A=(A \times[0,1]) /(\{*\} \times[0,1])$ be the cylinder in Top* 
Then the suspender $\Sigma_{f}$ of a map $f: A \rightarrow B$ is obtained by the pushout diagram

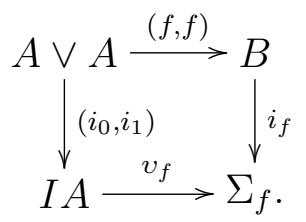

Here $v_{f}$ yields the track $v_{f}: i_{f} \Rightarrow i_{f}$ for the suspender $\Sigma_{f}$. Next let $P B=B^{I}$ be the space of maps $[0,1] \rightarrow B$ with the compact open topology. Then the looper $\Omega_{f}$ of $f$ is obtained by the pullback diagram

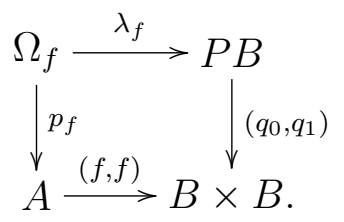

Here $\lambda_{f}$ yields the track $\lambda_{f}: p_{f} \Rightarrow p_{f}$ for the looper $\Omega_{f}$. In the next example we show that the properties 1.1 are satisfied for $\Sigma_{f}$ and $\Omega_{f}$ respectively.

Let $\mathbf{C}$ be a cofibration category in the sense of Baues [3]. For each cofibrant object $X$ in $\mathbf{C}$ we choose a cylinder

$$
X \vee X>I X \stackrel{\sim}{\longrightarrow} X
$$

which is a factorization of $(1,1): X \vee X \rightarrow X$. For a fibrant object $Y$ the homotopy classes relative to $X \vee X$ of maps $I X \rightarrow Y$ are the tracks in $\mathbf{C}$. Therefore the full subcategory $\mathbf{C}_{\mathrm{cf}}$ of cofibrant and fibrant objects in $\mathbf{C}$ is a track category; see [3, II $\S 5]$.

1.5. Lemma. For a cofibration category $\mathbf{C}$ the track category $\mathbf{C}_{\mathrm{cf}}$ is $\Sigma$-representable.

Proof. For each cofibrant object $X$ in $\mathbf{C}$ a fibrant model $j: X>\sim \sim R X$ can be chosen. Now the suspender $\Sigma_{f}$ of $f: A \rightarrow B$ in $\mathbf{C}_{\mathrm{cf}}$ is obtained by a fibrant model of the pushout $\Sigma_{f}^{\prime}$ in the following diagram

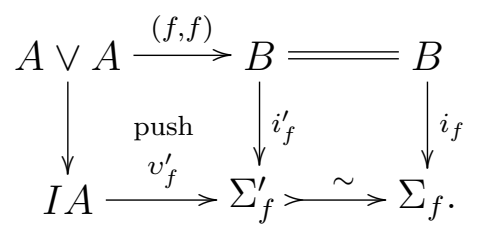

The composite $v_{f}=j v_{f}^{\prime}: I A \rightarrow \Sigma_{f}^{\prime} \rightarrow \Sigma_{f}$ yields the track $v_{f}: i_{f} \Rightarrow i_{f}$. We now check that the properties (a) and (b) in 1.1 are satisfied. For a map $g: B \rightarrow C$ in $\mathbf{C}_{\text {cf }}$ let $\eta: g f \simeq g f$ be a homotopy $\eta: I A \rightarrow C$. Then the pushout property of $\Sigma_{f}^{\prime}$ yields a map $g \cup \eta: \Sigma_{f}^{\prime} \rightarrow C$ which admits an extension $\Sigma_{\eta}: \Sigma_{f} \rightarrow C$ so that $\Sigma_{\eta} i_{f}=g$ and $\eta=\Sigma_{\eta} v_{f}$. Hence we can actually choose the track $\zeta_{\eta}$ in 
(a) to be the identity isomorphism of $g$. This proves (a). Now we check (b) as follows. Let $\gamma: h^{\prime} i_{f} \simeq h i_{f}$ with

$$
h^{\prime} v_{f}=\left(h v_{f}\right)^{\gamma f}=-\gamma f+h f+\gamma f
$$

as in (b). Here $(*)$ is an equation of tracks. Now $\left(^{*}\right)$ implies that there is a map

$$
\delta^{\prime}: I I A \rightarrow C
$$

with $\delta^{\prime} i_{0}=h v_{f}, \delta^{\prime} i_{1}=h^{\prime} v_{f}$ and $\delta^{\prime} I i_{0}=\delta^{\prime} I i_{1}=\gamma I f$. Here we choose the cylinder $I B$ to be a fibrant object so that $I f: I A \rightarrow I B$ is defined; see [3]. Now consider the following pushout diagram where $I(A \vee A)=I A \vee I A$.

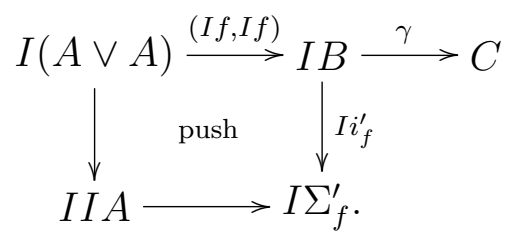

Here the pushout $I \Sigma_{f}^{\prime}$ is actually a cylinder for $\Sigma_{f}^{\prime}$ and we define a cylinder $I \Sigma_{f}$ for $\Sigma_{f}$ by the pushout diagram

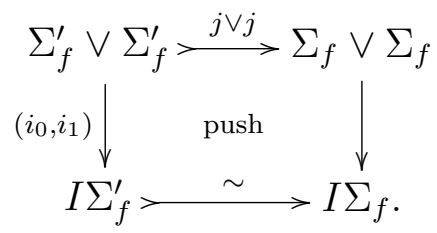

Now the map $\delta^{\prime} \cup \gamma: I \Sigma_{f}^{\prime} \rightarrow C$ is defined with $\left(\delta^{\prime} \cup \gamma\right) i_{0}=h^{\prime} j$ and $\left(\delta^{\prime} \cup \gamma\right) i_{1}=h j$. Hence a map $\delta=\left(\delta^{\prime} \cup \gamma\right) \cup\left(h^{\prime}, h\right): I \Sigma_{f} \rightarrow C$ is defined. The track defined by $\delta$ satisfies $\delta: h^{\prime} \Rightarrow h$. Moreover $\delta i_{f}=\gamma$ since $\delta i_{f}$ is represented by $\left(\delta^{\prime} \cup \gamma\right)\left(I i_{f}^{\prime}\right)=$ $\gamma$.

For a model category $\mathbf{Q}$ as in [11] let $\mathbf{Q}_{\mathrm{c}}$ and $\mathbf{Q}_{\mathrm{f}}$ denote the full subcategory of cofibrant and fibrant objects, respectively. Then $\mathbf{Q}_{c}$ is a cofibration category and $\mathbf{Q}_{\mathrm{f}}$ is a fibration category in the sense of Baues [3]. Here fibration category is the categorical dual of cofibration category. Therefore 1.5 above shows:

1.6. Corollary. Let $\mathbf{Q}_{\mathrm{cf}}$ be the track category of cofibrant and fibrant objects in a Quillen model category. Then $\mathbf{Q}_{\mathrm{cf}}$ is $\Sigma$-representable and $\Omega$-representable.

The examples in 1.4 are also consequences of 1.5 since Top* is a cofibration category and also a fibration category in which all objects are cofibrant and fibrant, compare [3]. Moreover using [3, Remark I.8.15] we see that also Top To $^{*}$ is a fibration category in which all objects are fibrant and cofibrant. Here we use the structure [3, I.3.3] and [3, I.4.6]. 


\section{FunCtorial PROPERTIES OF SUSPENDERS}

We consider functorial properties of suspenders. This implies a kind of uniqueness and compatibility with sums. For a category $\mathbf{T}$ the category $\operatorname{Pair}(\mathbf{T})$ is the usual category of pairs in $\mathbf{T}$. Objects of Pair $(\mathbf{T})$ are morphisms $A \rightarrow B$ and morphisms from $(A \rightarrow B)$ to $(X \rightarrow Y)$ are commutative diagrams in $\mathbf{T}$

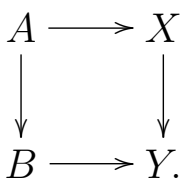

2.1. Lemma. For any commutative diagram of unbroken arrows

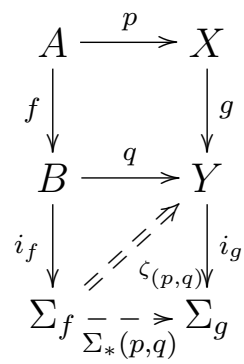

there exist a map $\Sigma_{*}(p, q)$ and a track $\zeta_{(p, q)}$ as indicated, with

$$
\Sigma_{*}(p, q) v_{f}=\left(v_{g} p\right)^{\zeta_{(p, q)} f} .
$$

Choosing such maps for each commutative square as above gives a functor

$$
\Sigma_{-}: \operatorname{Pair}(\mathscr{T}) \rightarrow \operatorname{Pair}(\mathscr{T} \simeq)
$$

carrying $f: A \rightarrow B$ to $\left[i_{f}\right] \in\left[B, \Sigma_{f}\right]$ and the commutative square $(p, q): f \rightarrow g$ as above to $\left([q],\left[\Sigma_{*}(p, q)\right]\right):\left[i_{f}\right] \rightarrow\left[i_{g}\right]$.

Proof. By the definition of suspenders, the track $v_{g} p$ considered as an automorphism of $i_{g} g p=i_{g} q f$ produces a map $\Sigma_{v_{g} p}: \Sigma_{f} \rightarrow \Sigma_{g}$ and a track $\zeta_{v_{g} p}: \Sigma_{v_{g} p} i_{f} \Rightarrow i_{g} q$ such that (a) holds. So one can define

$$
\Sigma_{*}(p, q)=\Sigma_{v_{g} p}, \quad \zeta_{(p, q)}=\zeta_{v_{g} p} .
$$

Then the injectivity condition for suspenders guarantees that there are tracks

$$
\Sigma_{*}\left(\operatorname{id}_{A}, \operatorname{id}_{B}\right) \simeq \operatorname{id}_{\Sigma_{f}}
$$

and

$$
\Sigma_{*}(p, q) \Sigma_{*}\left(p^{\prime}, q^{\prime}\right) \simeq \Sigma_{*}\left(p p^{\prime}, q q^{\prime}\right)
$$

for any two matching commutative squares. The lemma follows. 
2.2. Lemma. Let $\left(\Sigma_{f}, i_{f}, v_{f}\right)$ and $\left(\Sigma_{f}^{\prime}, i_{f}^{\prime}, v_{f}^{\prime}\right)$ be two suspenders of a map $f: A \rightarrow B$. Then they are equivalent in $\mathscr{T}$. More precisely, there exist maps $l: \Sigma_{f} \rightarrow \Sigma_{f}^{\prime}$ and $l^{\prime}: \Sigma_{f}^{\prime} \rightarrow \Sigma_{f}$ such that $l i_{f}=i_{f}^{\prime}, l^{\prime} i_{f}^{\prime}=i_{f}, l v_{f}=v_{f}^{\prime}$, and $l^{\prime} v_{f}^{\prime}=v_{f} . \quad$ Moreover there exist tracks $\lambda: l^{\prime} l \simeq \operatorname{id}_{\Sigma_{f}}, \lambda^{\prime}: l l^{\prime} \simeq \operatorname{id}_{\Sigma_{f}^{\prime}}$ with $\lambda i_{f}=\operatorname{id}_{i_{f}}, \lambda^{\prime} i_{f}^{\prime}=\operatorname{id}_{i_{f}^{\prime}}$.

Proof. Existence of $l=\Sigma_{v_{f}^{\prime}}$ and $l^{\prime}=\Sigma_{v_{f}}$ satisfying the required identities is clear from the definition of suspenders. Then further by the uniqueness property of suspenders, for the identity track $\Sigma_{v_{f}^{\prime}} \Sigma_{v_{f}} i_{f}=\Sigma_{v_{f}^{\prime}} i_{f}^{\prime}=i_{f}=\mathrm{id}_{\Sigma_{f}} i_{f}$ one has $\Sigma_{v_{f}^{\prime}} \Sigma_{v_{f}} v_{f}=\Sigma_{v_{f}^{\prime}} v_{f}^{\prime}=v_{f}$, hence there is a track $\lambda: \Sigma_{v_{f}^{\prime}} \Sigma_{v_{f}} \simeq \operatorname{id}_{\Sigma_{f}}$ with $\lambda i_{f}=\mathrm{id}_{i_{f}}$. In an exactly symmetric way one has $\lambda^{\prime}$ with required properties.

Also the converse is true:

2.3. Lemma. Let $\left(\Sigma_{f}, i_{f}, v_{f}\right)$ be a suspender for the map $f: A \rightarrow B$ and let the maps $l: \Sigma_{f} \rightarrow \Sigma$, $l^{\prime}: \Sigma \rightarrow \Sigma_{f}$ and tracks $\lambda: l^{\prime} l \simeq \operatorname{id}_{\Sigma_{f}}, \lambda^{\prime}: l l^{\prime} \simeq \operatorname{id}_{\Sigma}$ realise a homotopy equivalence. Then $\left(\Sigma, l i_{f}, l v_{f}\right)$ is another suspender for $f$.

Proof. Consider the composite functor

$$
\llbracket \Sigma,-\rrbracket \stackrel{\llbracket l,-\rrbracket}{\longrightarrow} \llbracket \Sigma_{f},-\rrbracket \stackrel{\left(i_{f}, v_{f}\right)^{*}}{\longrightarrow} \mathbf{G}_{f}
$$

Clearly it coincides with $\left(l i_{f}, l v_{f}\right)^{*}$. Moreover $\left(i_{f}, v_{f}\right)^{*}$ induces bijection on isomorphism classes by the universal property of suspenders, and so does $\llbracket l,-\rrbracket$ - in fact the latter is an equivalence, with inverse $\llbracket l^{\prime},-\rrbracket$. Hence the lemma.

2.4. Lemma. For any object $A$, a suspender for the map $!_{A}: * \rightarrow$ A from the (possibly weak) initial object to $A$ is given by $\left(A, \mathrm{id}_{A}, \mathrm{id}_{!_{A}}\right)$. Given suspenders $\left(\Sigma_{f}, i_{f}, v_{f}\right)$ and $\left(\Sigma_{f^{\prime}}, i_{f^{\prime}}, v_{f^{\prime}}\right)$ for the maps $f: A \rightarrow B$ and $f^{\prime}: A^{\prime} \rightarrow B^{\prime}$, respectively, $\left(\Sigma_{f} \vee \Sigma_{f^{\prime}}, i_{f} \vee i_{f^{\prime}}, v_{f} \vee v_{f^{\prime}}\right)$ is a suspender for $f \vee f^{\prime}: A \vee A^{\prime} \rightarrow B \vee B^{\prime}$.

Proof. The first assertion follows easily from the fact that the functor $\mathbf{G}_{!_{A}}$ coincides with the covariant representable functor $\llbracket A,-\rrbracket$.

For the second, consider the functors

$$
\llbracket \Sigma_{f} \vee \Sigma_{f^{\prime}},-\rrbracket \stackrel{\simeq}{\lessgtr} \llbracket \Sigma_{f},-\rrbracket \times \llbracket \Sigma_{f^{\prime}},-\rrbracket \stackrel{\left(i_{f}, v_{f}\right)^{*} \times\left(i_{f^{\prime}}, v_{f^{\prime}}\right)^{*}}{\longrightarrow} \mathbf{G}_{f} \times \mathbf{G}_{f^{\prime}} \rightarrow \mathbf{G}_{f \vee f^{\prime}}
$$

where the rightmost functor is the one assigning to $\left((g, \alpha),\left(g^{\prime}, \alpha^{\prime}\right)\right)$ with $g$ : $B \rightarrow C, \alpha: g f \simeq g f, g^{\prime}: B^{\prime} \rightarrow C^{\prime}, \alpha^{\prime}: g^{\prime} f^{\prime} \simeq g^{\prime} f^{\prime}$ the pair $\left(\left(\begin{array}{c}g \\ g^{\prime}\end{array}\right),\left(\begin{array}{c}\alpha \\ \alpha^{\prime}\end{array}\right)\right)$, where $\left(\begin{array}{c}g \\ g^{\prime}\end{array}\right): B \vee B^{\prime} \rightarrow C$ and $\left(\begin{array}{c}\alpha \\ \alpha^{\prime}\end{array}\right):\left(\begin{array}{c}g f \\ g^{\prime} f^{\prime}\end{array}\right) \simeq\left(\begin{array}{c}g f \\ g^{\prime} f^{\prime}\end{array}\right) \simeq\left(\begin{array}{c}g \\ g^{\prime}\end{array}\right)\left(f \vee f^{\prime}\right)$ are obtained from the equivalences $\llbracket B \vee B^{\prime}, C \rrbracket \simeq \llbracket B, C \rrbracket \times \llbracket B^{\prime}, C \rrbracket$. It is clear how to define this functor on morphisms. One sees directly that this functor induces bijection on isomorphism classes of objects; hence so does the composite, which is easily seen to coincide with $\left(i_{f} \vee i_{f^{\prime}}, v_{f} \vee v_{f^{\prime}}\right)^{*}$. The lemma follows. 


\section{Suspensions}

Let $*$ be the initial object of a track category $\mathscr{T}$ in the strong sense so that the hom-groupoid $\llbracket *, X \rrbracket$ is the trivial groupoid for any $X$. Then the suspender $\Sigma_{0} A$ of a map $0: A \rightarrow *$ is termed a suspension (associated to 0 ) of $A$.

3.1. Proposition. For any map $0: A \rightarrow *$ to the initial object, the corresponding suspension $\Sigma_{0}(A)$ is canonically equipped with a cogroup structure in the homotopy category $\mathscr{T}_{\simeq}$. Moreover for any $a: A^{\prime} \rightarrow A$ the induced map (see 2.1) $\Sigma_{*}\left(f, \mathrm{id}_{*}\right): \Sigma_{0 a}(A) \rightarrow \Sigma_{0}(A)$ respects this cogroup structure.

Proof. Recall that the initial object is understood in the strong sense, so that $\llbracket *, X \rrbracket$ is a trivial groupoid for any $X$. It then follows that the groupoid $\mathbf{G}_{0}(X)$ has as many objects as there are tracks $\alpha: !_{X} 0 \simeq !_{X} 0$, and only identity morphisms. In other words, it is the discrete groupoid on the set Aut $\left(!_{X} 0\right)$. Let us equip this set with a group structure coming from the obvious one on Aut $\left(!_{X} 0\right)$. Then moreover the functor $\mathbf{G}_{0}(X) \rightarrow \mathbf{G}_{0}(Y)$ induced by a map $f: X \rightarrow Y$ is given on objects by $\alpha \mapsto f \alpha$, hence is a homomorphism of groups. One so obtains a lifting of the functor $\mathbf{G}_{0}$ to groups. But by the universal property of the suspender, this functor coincides with $\left[\Sigma_{0},-\right]$. So considered as an object of $\mathscr{T} \simeq$, the suspension $\Sigma_{0}$ has the property that its covariant representable functor lifts to the category of groups. It then follows by the standard categorical argument that this object has a cogroup structure in $\mathscr{T}$. Explicitly, the cozero of this cogroup is $\Sigma_{\mathrm{id}_{0}}$, i. e. the map $\Sigma_{0} \rightarrow *$ induced by the pair $\left(\mathrm{id}_{*}: * \rightarrow *, \mathrm{id}_{0}: 0 \mathrm{id}_{*}=0 \simeq 0=0 \mathrm{id}_{*}\right)$. The coaddition map $+: \Sigma_{0} \rightarrow \Sigma_{0} \vee \Sigma_{0}$ is induced by the pair $\left(!_{\Sigma_{0} \vee \Sigma_{0}}: * \rightarrow \Sigma_{0} \vee \Sigma_{0}, i_{1} v_{0}+i_{2} v_{0}\right)$, where $v_{0} \in \operatorname{Aut}\left(!_{\Sigma_{0}} 0\right)$ is the universal track and $i_{1}, i_{2}: \Sigma_{0} \rightarrow \Sigma_{0} \vee \Sigma_{0}$ are the coproduct inclusions. The inverse map $\Sigma_{0} \rightarrow \Sigma_{0}$ is induced by $\left(!_{\Sigma_{0}} 0,-v_{0}\right)$.

Now given any $a: A^{\prime} \rightarrow A$, it obviously respects counit. To show that it respects coaddition, one must find a track $+\Sigma_{*}\left(a, \operatorname{id}_{*}\right) \simeq\left(\Sigma_{*}\left(a, \operatorname{id}_{*}\right) \vee \Sigma_{*}\left(a, \operatorname{id}_{*}\right)\right)+$. According to the uniqueness property of the suspender $\Sigma_{0 a}$, for this it is enough to find a track $\alpha:+\Sigma_{*}\left(a, \mathrm{id}_{*}\right) i_{0 a} \simeq\left(\Sigma_{*}\left(a, \mathrm{id}_{*}\right) \vee \Sigma_{*}\left(a, \mathrm{id}_{*}\right)\right)+i_{0 a}$ satisfying $+\Sigma_{*}\left(a, \mathrm{id}_{*}\right) v_{0 a}=\left(\left(\Sigma_{*}\left(a, \mathrm{id}_{*}\right) \vee \Sigma_{*}\left(a, \mathrm{id}_{*}\right)\right)+v_{0 a}\right)^{\alpha 0 a}$. There is a unique choice for such $\alpha$ - namely the identity track, as $*$ is initial in the strong sense. Then $+\Sigma_{*}\left(a, \mathrm{id}_{*}\right) v_{0 a}=+v_{0} a=\left(i_{1} v_{0}+i_{2} v_{0}\right) a=i_{1} v_{0} a+i_{2} v_{0} a=i_{1} \Sigma_{*}\left(a, \mathrm{id}_{*}\right) v_{0 a}+$ $i_{2} \Sigma_{*}\left(a, \mathrm{id}_{*}\right) v_{0 a}=\left(\left(\Sigma_{*}\left(a, \mathrm{id}_{*}\right) \vee \Sigma_{*}\left(a, \mathrm{id}_{*}\right)\right) i_{1} v_{0 a}+\left(\Sigma_{*}\left(a, \mathrm{id}_{*}\right) \vee \Sigma_{*}\left(a, \mathrm{id}_{*}\right)\right) i_{2} v_{0 a}\right.$ $=\left(\Sigma_{*}\left(a, \mathrm{id}_{*}\right) \vee \Sigma_{*}\left(a, \mathrm{id}_{*}\right)\right)\left(i_{1} v_{0 a}+i_{2} v_{0 a}\right)=\left(\Sigma_{*}\left(a, \operatorname{id}_{*}\right) \vee \Sigma_{*}\left(a, \mathrm{id}_{*}\right)\right)+v_{0 a}$ as required.

3.2. Corollary. Suppose that an object $A$ has a co-H-structure, i. e. a coaddition $a: A \rightarrow A \vee A$ with a two-sided cozero $0: A \rightarrow *$ in $\mathscr{T}_{\simeq}$. Then the above canonical cogroup structure on $\Sigma_{0}$ (see 3.1) is coabelian.

Proof. By 2.1 and 2.4, there are maps $0^{\prime}=\Sigma_{*}\left(0, \mathrm{id}_{*}\right): \Sigma_{0} \rightarrow *$ and $+^{\prime}=$ $\Sigma_{*}\left(a, \mathrm{id}_{*}\right): \Sigma_{0} \rightarrow \Sigma_{0} \vee \Sigma_{0}$ which equip $\Sigma_{0}$ with a co- $H$-structure in $\mathscr{T}$. On the other hand it has a canonical cogroup structure $\left(\Sigma_{\mathrm{id}_{0}},+,-\right)$ in $\mathscr{T} \simeq$ by 3.1 . 
But in fact $\Sigma_{\text {id }_{0}}$ and $\Sigma_{*}\left(0, \mathrm{id}_{*}\right)$ coincide in $\mathscr{T} \simeq$, so it follows that these cogroup structures have the same cozero.

Moreover the fact that $+^{\prime}$ respects the cogroup structure means commutativity of

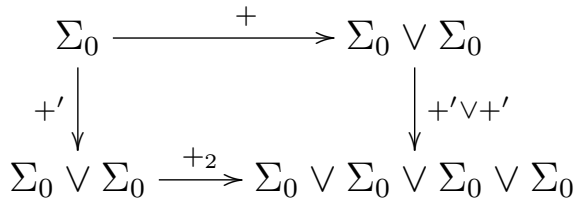

in $\mathscr{T}$, where $+_{2}$ is the coaddition for the canonical cogroup structure on $\Sigma_{0} \vee \Sigma_{0}$ considered as $\Sigma_{\left(\begin{array}{l}0 \\ 0\end{array}\right)}$. It is clear that this cogroup structure coincides with the coproduct of cogroup structures on $\Sigma_{0}$. In general, for two cogroups $X$ and $Y$ the coaddition on their coproduct is given by

$$
X \vee Y \stackrel{+_{X} \vee+_{Y}}{\longrightarrow} X \vee X \vee Y \vee Y \stackrel{X \vee\left(\begin{array}{l}
i_{X} \\
i_{Y}
\end{array}\right) \vee Y}{\longrightarrow} X \vee Y \vee X \vee Y
$$

so that $+_{2}$ is given by the composite

$$
\Sigma_{0} \vee \Sigma_{0} \stackrel{+\vee+}{\longrightarrow} \Sigma_{0} \vee \Sigma_{0} \vee \Sigma_{0} \vee \Sigma_{0} \stackrel{(23)}{\longrightarrow} \Sigma_{0} \vee \Sigma_{0} \vee \Sigma_{0} \vee \Sigma_{0}
$$

where (23) denotes the map permuting second and third summands.

Composing the above diagram with

$$
\operatorname{id}_{\Sigma_{0}} \vee\left(\begin{array}{c}
0^{\prime} \\
0^{\prime}
\end{array}\right) \vee \operatorname{id}_{\Sigma_{0}}: \Sigma_{0} \vee \Sigma_{0} \vee \Sigma_{0} \vee \Sigma_{0} \rightarrow \Sigma_{0} \vee * \vee * \vee \Sigma_{0} \cong \Sigma_{0} \vee \Sigma_{0}
$$

then gives that there is a track $+^{\prime} \simeq+$, whereas composing it with

$$
\left(\begin{array}{l}
0^{\prime} \vee \operatorname{id}_{\Sigma_{0}} \\
\operatorname{id}_{\Sigma_{0}} \vee 0^{\prime}
\end{array}\right): \Sigma_{0} \vee \Sigma_{0} \vee \Sigma_{0} \vee \Sigma_{0} \rightarrow * \vee \Sigma_{0} \vee \Sigma_{0} \vee * \cong \Sigma_{0} \vee \Sigma_{0}
$$

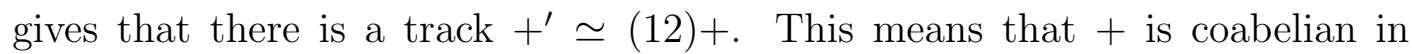
$\mathscr{T}$.

\section{Suspenders of COACTIONS}

We show that the suspender $\Sigma_{f}$ of a map $f: A \rightarrow B$ splits as a coproduct if $A$ has the structure of a principal coaction in the homotopy category. Recall that a theory $\mathbf{C}$ is a category with finite sums $A \vee B$.

4.1. Definition. A principal coaction in a theory $\mathbf{C}$ consists of an object $A$ together with a cogroup object $S$ in $\mathbf{C}$ and a right coaction

$$
a: A \rightarrow A \vee S
$$

on $A$ such that the map

$$
\left(i_{A}, q\right): A \vee A \rightarrow A \vee S
$$

is an isomorphism in $\mathbf{C}$. The cogroup structure of $S$ is given by maps $\mu: S \rightarrow$ $S \vee S, \nu: S \rightarrow S$ and $e: S \rightarrow *$. The inverse of $\left(i_{A}, a\right)$ yields the map $d: S \rightarrow A \vee A$. 
A principal coaction is trivial if $A$ is isomorphic to $S$ in such a way that $\mu$ corresponds to the cogroup structure of $S$. It is well known that a principal coaction $(A, a)$ is trivial if and only if there exists a map $A \rightarrow *$ in $\mathbf{C}$, where * is the initial object of $\mathbf{C}$.

4.2. Remark. A principal action in a category with finite products consists of an object $T$ together with an internal group $G$ in this category and a right action $a: T \times G \rightarrow T$ of $G$ on $T$ such that the map $\left(p_{T}, a\right): T \times G \rightarrow T \times T$ is an isomorphism ( $p_{T}$ being the product projection). Of course a principal action is the categorical dual of a principal coaction.

For our purposes we need a weaker notion which we call principal quasi action or quasi torsor. It consists of objects $T, G$ and morphisms $T \times G \rightarrow T, d$ : $T \times T \rightarrow G, 1 \rightarrow G$, denoted via $(x, g) \mapsto x \cdot g,(x, y) \mapsto x \backslash y$, and $e$ respectively, for $x, y: ? \rightarrow T, g: ? \rightarrow G$, such that the following identities hold:

- $x \backslash x=e$;

- $x \cdot(x \backslash y)=y$.

(Note that the above conditions imply also $x \cdot e=x$.)

Clearly, any principal action is a particular case of this, as one can define $d$ to be the composite map

$$
T \times T \stackrel{\left(p_{T}, a\right)^{-1}}{\longrightarrow} T \times G \stackrel{p_{G}}{\longrightarrow} G .
$$

The categorical dual of a principal quasi action is a principal quasi coaction which generalizes the principal coaction in 4.1.

Recall that a track theory is a track category with coproducts $A \vee B$ in the weak sense (see [5]) so that for all $X$ one has the equivalence of hom-groupoids

$$
\llbracket A \vee B, X \rrbracket \stackrel{\sim}{\longrightarrow} \llbracket A, X \rrbracket \times \llbracket B, X \rrbracket .
$$

Let $\omega$ be an inverse of this equivalence.

4.3. Theorem. Let $\mathscr{T}$ be a track theory and let $f: A \rightarrow B$ be a map in $\mathscr{T}$. Assume $A$ has the structure of a principal (quasi)coaction in the homotopy category $\mathscr{T} \simeq$ represented by a map $a: A \rightarrow A \vee S$ in $\mathscr{T}$, where $S$ is a cogroup in $\mathscr{T}_{\simeq}$. Let $\Sigma S=\Sigma_{e} S$ be a suspension of $S$ in $\mathscr{T}$ associated to a map $e: S \rightarrow *$ in $\mathscr{T}$ representing the counit of $S$. Then there is a suspender of $f$ with

$$
\Sigma_{f}=B \vee \Sigma S
$$

and $i_{f}=i_{B}: B \rightarrow B \vee \Sigma S$ the coproduct inclusion and $v_{f}: i_{B} f \Rightarrow i_{B} f$ a certain canonically defined track.

The theorem shows that existence of certain suspensions in a track category implies existence of a wider class of suspenders. Moreover by 2.2 we get the following corollary. 
4.4. Corollary. Let $\mathscr{T}$ be a $\Sigma$-representable track theory and let $f: A \rightarrow B$ be a map in $\mathscr{T}$ where $A$ admits the structure of a principal (quasi)coaction $A \rightarrow A \vee S$ in $\mathscr{T}$. Then there exists a homotopy equivalence $\Sigma_{f} \simeq B \vee \Sigma S$ where $\Sigma S$ is a suspension associated to a map $S \rightarrow *$ representing the counit of $S$.

Proof of 4.3. To simplify exposition, let us introduce the following notation. The given principal coaction gives rise, for each object $X$, to functors

$$
\llbracket A, X \rrbracket \times \llbracket S, X \rrbracket \stackrel{\omega}{\longrightarrow} \llbracket A \vee S, X \rrbracket \stackrel{\llbracket a, X \rrbracket}{\longrightarrow} \llbracket A, X \rrbracket
$$

and

$$
\llbracket A, X \rrbracket \times \llbracket A, X \rrbracket \stackrel{\omega}{\longrightarrow} \llbracket A \vee A, X \rrbracket \stackrel{\llbracket d, X \rrbracket}{\longrightarrow} \llbracket S, X \rrbracket,
$$

whose actions on both objects and morphisms will be denoted by

$$
(x, s) \mapsto a \cdot s, \quad(x, y) \mapsto x \backslash y,
$$

respectively. The principal coaction structure in $\mathscr{T} \simeq$ implies existence of tracks $\varkappa, \lambda$ which for any $x, y: A \rightarrow X$ induce tracks

$$
\begin{gathered}
x \varkappa: e \Rightarrow x \backslash x, \\
\left(\begin{array}{l}
x \\
y
\end{array}\right) \lambda: x \cdot(x \backslash y) \Rightarrow y .
\end{gathered}
$$

Let us define another track $\iota$ by

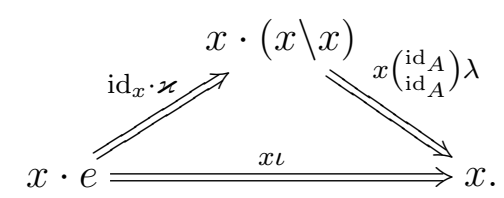

We now turn to the construction of the universal track $v_{f}$. It is the composite track in the diagram

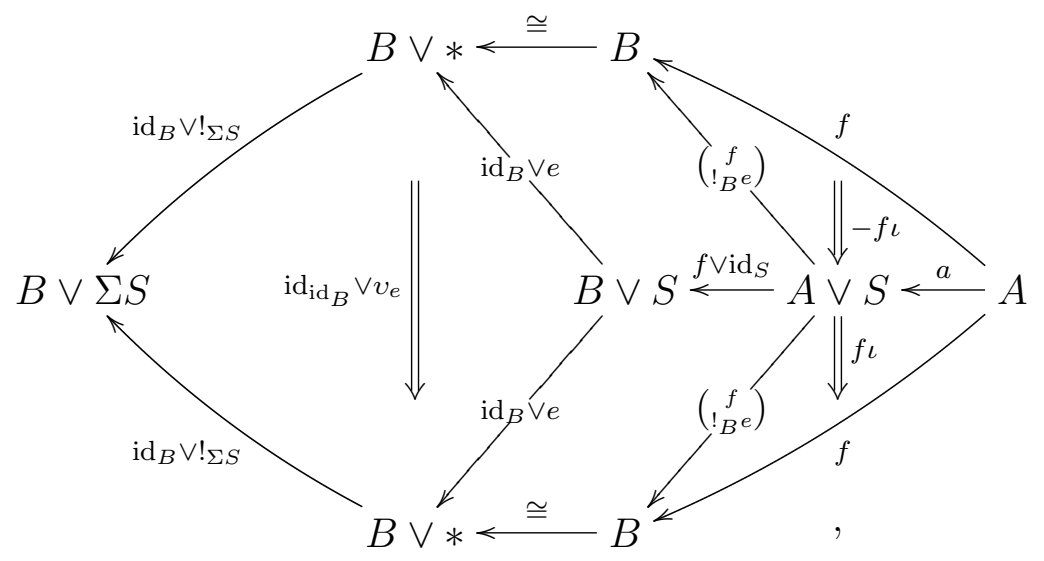

where the two parallelograms commute. More formally, $v_{f}=(v a)^{-i_{B} f \iota}$, where the track

$$
v=\left(\operatorname{id}_{\mathrm{id}_{B}} \vee v_{e}\right)\left(f \vee \mathrm{id}_{S}\right) \in \operatorname{Aut}\left(\left(\operatorname{id}_{B} \vee\left(!_{\Sigma S} e\right)\right)\left(f \vee \mathrm{id}_{S}\right)\right)
$$


is considered as an automorphism of the map

$$
\begin{aligned}
& i_{B}\left(\begin{array}{c}
f \\
!_{B} e
\end{array}\right)=\left(\operatorname{id}_{B} \vee !_{\Sigma S}\right)(f \vee e) \\
= & \left(\operatorname{id}_{B} \vee !_{\Sigma S}\right)\left(\operatorname{id}_{B} \vee e\right)\left(f \vee \operatorname{id}_{S}\right) \\
= & \left(\operatorname{id}_{B} \vee\left(!_{\Sigma S} e\right)\right)\left(f \vee \operatorname{id}_{S}\right) .
\end{aligned}
$$

To show that $v_{f}$ is indeed universal, we must show that, for each object $X$, the functor

$$
\llbracket B, X \rrbracket \times \llbracket \Sigma S, X \rrbracket \cong \llbracket B \vee \Sigma S, X \rrbracket \stackrel{\left(i_{B}, v_{f}\right)^{*}}{\longrightarrow} \mathbf{G}_{f}(X)
$$

induces bijection on isomorphism classes of objects. Now $v_{f}$ is chosen in such a way that given $x: B \rightarrow X$ and a track $\varepsilon \in \operatorname{Aut}\left(!_{X} e\right)$ with the corresponding $\operatorname{map} \Sigma_{\varepsilon}: \Sigma S \rightarrow X$, one has

$$
\left(i_{B}, v_{f}\right)^{*}\left(x, \Sigma_{\varepsilon}\right)=\left(\operatorname{id}_{x f} \cdot \varepsilon\right)^{-x f \iota}: x f \simeq x f .
$$

Taking into account the universal property of $\Sigma S$, we may replace isomorphism classes of $\llbracket \Sigma S, X \rrbracket$ by those of $\mathbf{G}_{e}(X)$. We thus must show

- For any $x: B \rightarrow X$ and any track $\alpha \in \operatorname{Aut}(x f)$ there is a track $\varepsilon \in$ Aut $\left(!_{X} e\right)$ such that $\operatorname{id}_{x f} \cdot \varepsilon=\alpha^{x f \iota}$;

- For any $x, x^{\prime}: B \rightarrow X$, any $\varepsilon, \varepsilon^{\prime} \in \operatorname{Aut}\left(!_{X} e\right)$ and any $\chi: x \simeq x^{\prime}$ with $\operatorname{id}_{x^{\prime} f} \cdot \varepsilon^{\prime}=\left(\operatorname{id}_{x f} \cdot \varepsilon\right)^{\chi f}$ there is a track $\eta: \Sigma_{\varepsilon^{\prime}} \rightarrow \Sigma_{\varepsilon}$.

For the first, define, for $\alpha \in \operatorname{Aut}(x f)$, the track $\varepsilon=\left(\operatorname{id}_{x f} \backslash \alpha\right)^{x f \varkappa}$. Then because of our special choice of $\iota$ the required identity will be satisfied.

For the second, note that if $\chi: x \simeq x^{\prime}$ satisfies the hypothesis, then in the diagram

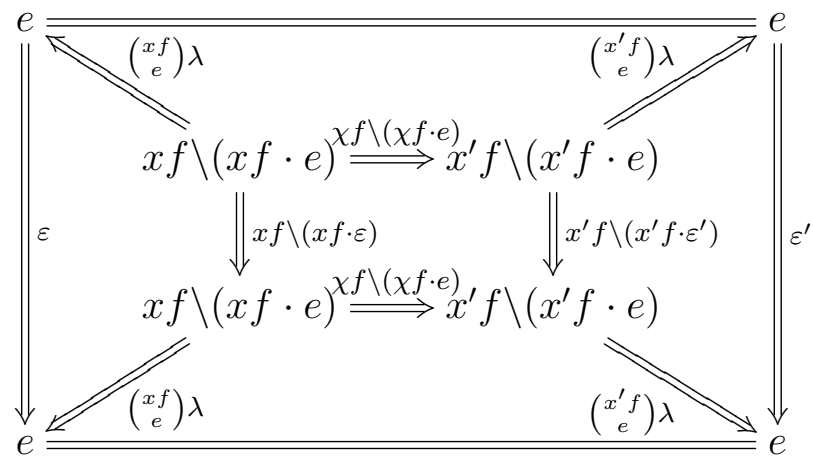

all inner squares commute, hence the outer square commutes too, i. e. actually $\varepsilon=\varepsilon^{\prime}$.

\section{ACKNOWLEDGEMENT}

The second author gratefully acknowledges hospitality of the Max Planck Institut für Mathematik, Bonn and of the Université Catholique de Louvain, Louvain-la-Neuve. 


\section{REFERENCES}

1. W. D. Barcus and M. G. Barratt, On the homotopy classification of the extensions of a fixed map. Trans. Amer. Math. Soc. 88(1958), 57-74.

2. H.-J. Baues, Obstruction theory. Lecture Notes in Math., 628, Springer, Berlin-N. Y. 1977.

3. H.-J. Baues, Algebraic homotopy. Cambridge studies in advanced mathematics, 15, Cambridge University Press, Cambridge 1989.

4. H.-J. BAues, On the cohomology of categories, universal Toda brackets and homotopy pairs. K-theory 11(1997), 259-285.

5. H.-J. Baues and M. Jibladze, Classification of abelian track categories. Preprint.

6. H.-J. Baues and M. Jibladze, Suspension and loop objects in theories and cohomology. Georgian Math. J. 8(2001), No. 4, 697-712.

7. P. Gabriel and M. Zisman, Calculus of fractions and homotopy theory. Ergebnisse der Math. und ihrer Grenzgebiete 35, Springer, N. Y. 1967.

8. K. A. Hardie, K. H. Kamps and R. W. Kieboom, A homotopy category of maps and tracks. Preprint.

9. K. A. Hardie, K. H. Kamps and R. W. Kieboom, A homotopy bigroupoid of a topological space. Preprint.

10. K. A. Hardie, H. J. Marcum and N. Oda, Bracket operations in the homotopy theory of a 2-category. Preprint.

11. D. G. Quillen, Homotopical algebra. Lecture Notes in Math. 43 Springer, Berlin-N. Y. 1967.

12. J. W. Rutter, A homotopy classification of maps into an induced fibre space. Topology 6(1967), 379-403.

(Received 28.11.2000)

Authors' addresses:

H.-J. Baues

Max-Planck-Institut fuer Mathematik

Vivatsgasse 7, D-53111 Bonn

Germany

E-mail: baues@mpim-bonn.mpg.de

M. Jibladze

A. Razmadze Mathematical Institute Georgian Academy of Sciences

1, M. Aleksidze St., Tbilisi 380093

Georgia

E-mail: jib@rmi.acnet.ge 